\title{
Design Optimisation of Façade-Integrated Photobioreactors Using CFD Simulation
}

\author{
Jake Haskell $^{1}$, Matthias Frechen ${ }^{1}$, Timo Sengewald ${ }^{1}$ \\ ${ }^{1}$ Advanced Building Engineering, ARUP, Berlin, Germany
}

\begin{abstract}
Following the success of the BIQ Haus in 2013, improved photobioreactors are being developed for façade integration in buildings. The photobioreactor façade panels, in which microalgae biomass is cultivated, also act as solar collectors and generate energy for building occupants. Algae growth in the reactors is positively influenced by high velocities which transport microalgae cells to the reactor surface more frequently, allowing them to absorb more sunlight and grow more efficiently. This paper outlines CFD flow simulations of the photobioreactors conducted to investigate the effects of different reactor geometries on flow velocities and improve the efficiency of next generation reactor panels.
\end{abstract}

\section{Introduction}

\section{Background}

In 2013, the BIQ House was completed as part of the International Building Exhibition in Hamburg and became the first building in the world with photobioreactor elements on its façade (see figure 1).

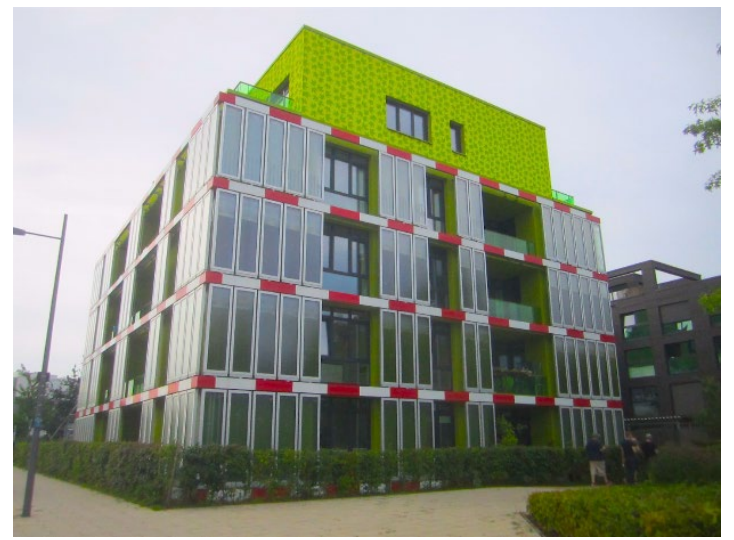

Figure 1: BIQ Haus in Hamburg with photobioreactor façade

Based on the experience gained during the implementation and operation of the BIQ Haus, the photobioreactors (PBRs) are now being further developed as part of an interdisciplinary research project to design optimised façade panels with improved efficiency which are also thinner, lighter and easier to manufacture, operate and maintain.

\section{Photobioreactor Panels}

Microalgae are cultivated in the PBRs on the façade of the building and provide a continuous source of renewable biomass which can be used as a valuable nutrient for processing into food or biofuel. The growth of this biomass also binds carbon dioxide from the atmosphere, which makes the use of photobioreactors as a 'green' technology particularly attractive. In addition to the biomass production, the PBRs also act as solar thermal collectors for the building with the algae fluid as the carrier medium. In tandem with the photosynthetic growth processes of the microalgae occurring in the reactors, the thermal energy harvested in the PBRs from the sun is utilized by the building. The heat is transferred from the PBRs via heat exchangers to a heat pump to provide a portion of the energy required for hot water and heating. An automated process regularly harvests algae when the carrier medium has reached a certain biomass density. The entire system thus represents an active and dynamic façade that is strongly linked to the building's operation and energy supply. The PBR façade panels serve as a regenerative and decentralised energy system for the building.

The photobioreactors are integrated into facade panels (elements) such that the microalgae are cultivated in a $10 \mathrm{~mm}$ thin fluid layer between two panes of composite safety glass. To grow efficiently and effectively in the thin reactor layer, the microalgae cells should be transported away from the sun-irradiated surface frequently as they cannot absorb further photons for 20$50 \mathrm{~ms}$ after light exposure. Thus a rapid and consistent transport of the microalgae to and from the irradiated surface is desirable and positive for efficient growth (Leupold et al. 2013).

In order to increase the frequency with which microalgae cells move from the front to the back of the reactor, the algae fluid in each façade-integrated photobioreactor is mixed using periodic injections of compressed air (airlift mixing). The air is injected into the reactor bottom via valves in short, periodic intervals, so that the injection impulse and the rising air bubbles that form ensure sufficient circulation in the algae fluid and transverse transport of the algae cells. An image of an ascending air bubble in the reactor as a result of an air injection is shown in figure 2 .

The aim is to achieve the highest possible flow velocities in the algae fluid phase in the PBR with the lowest 
possible energy consumption for the compressed air injections to increase the overall efficiency of the system (Hindersin et al., 2013), (Leupold et al. 2013). Dynamic flow simulations are performed for various reactor designs to assess the magnitude of the fluid velocities during operation with the goal of identifying a reactor design that favours higher flow velocities as well as a high static robustness while remaining simple and relatively light.

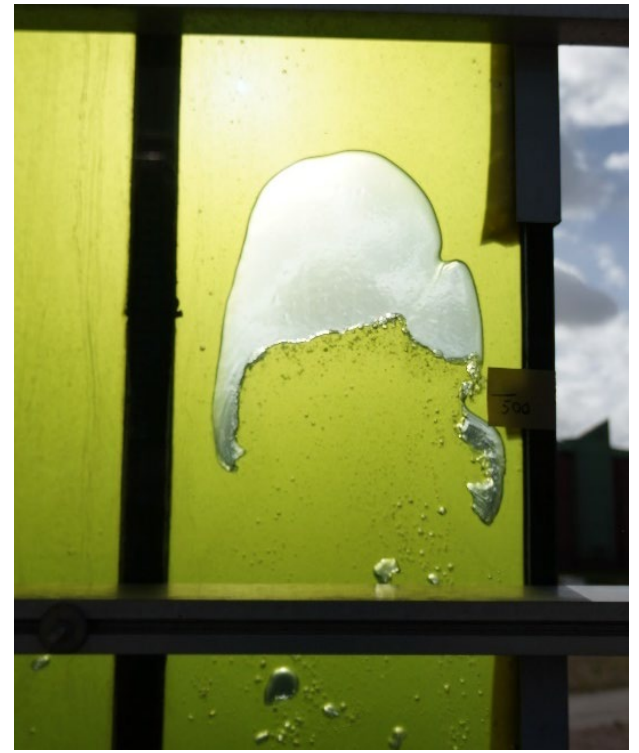

Figure 2: Airlift bubble rising in channel of reactor prototype

\section{Concept}

The algae need solar radiation and carbon dioxide to grow during the photosynthetic process. In order to achieve higher yields and accelerate the growth rate, the microalgae also need a certain amount of mixing to ensure better light distribution of the incident radiation to the individual algae cells in the reactor (Hindersin et al., 2013), (Leupold et al. 2013).

This airlift mixing in the reactor (see overview in figure 3 ), ensures that velocities in the algae fluid remain as high as possible. In addition, the rising air bubbles elicit further movement of the algae fluid medium in the reactor. The entire system represents a two-phase mixture of air and algae fluid with strongly transient characteristics, which is modelled with the help of threedimensional CFD simulations.

Figure 3 shows a schematic of the reactor model with the operation cycles for the airlift injections. In order to keep the energy required for airlift mixing low and to protect the reactor from excessive pressure fluctuations, air is injected into only two of the four reactor channels during each airlift cycle. The airlift is executed in alternate cycles for the channels $1+3$ and $2+4$ according to the specified interval duration of the air injection (see figure $3)$.

During each airlift cycle $500 \mathrm{~cm}^{3}$ of compressed air is injected into 2 of the 4 channels for 0.5 seconds through a valve at the bottom of the reactor. An airlift in the system occurs every 4 seconds so that each channel receives an injection of air every 8 seconds (see figure 3 ). The air pumped into to the reactor rises and escapes through small openings in the top of the reactor.

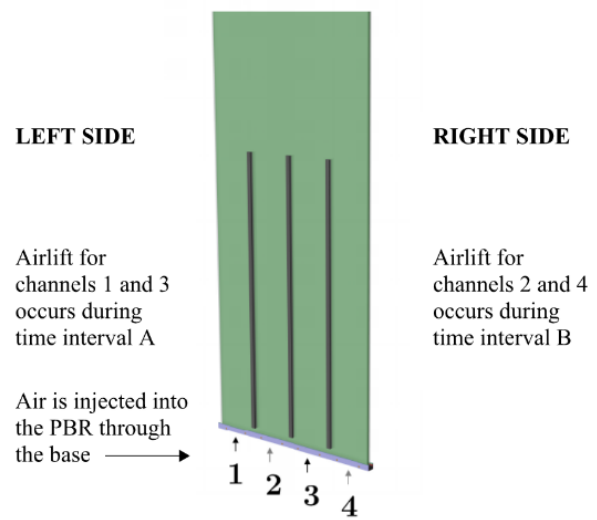

Figure 3: Airlift injection cycles in the photobioreactor

\section{Methods}

The CFD software OpenFOAM is used to simulate the flow in selected reactor variants (Greenshields 2014), (Greenshields 2015). The evaluation of the simulation results is performed with the data visualization software Paraview as well as Excel and Python for data analysis. Prior to simulation, a detailed review of relevant literature on the modelling of two-phase flows in reactors and bubble columns was carried out. See Becker et al. 1999, Clift et al. 1978, Hill 1998, Marschall 2011, Ratkovich 2009, Rusche 2002, Wang 2015 and Ziegenhein et al. 2014.

The PBR, which is integrated into a façade element panel, has a fixed height of $3.00 \mathrm{~m}$ and a fixed width of $1.35 \mathrm{~m}$. The depth of the reactor (between the glass panes) filled with algae fluid is $10 \mathrm{~mm}$ and the reactor is filled to $\quad 2.80 \mathrm{~m}$. While the external dimensions of the reactor are determined by the dimensions of the facade element and as such fixed, the length and width of the necessary structural support elements in the reactor, the so-called 'dividers', are variable. The integration of the dividers into the reactor design results in four channels, each approximately $32 \mathrm{~cm}$ wide.

As the goal of the analysis is to investigate the velocities in the entire reactor with reasonable calculation times, the Euler-Euler approach (Volume-of-Fluid method, $\mathrm{VoF})$ is utilized. A more precise method of modelling the two-phase dynamics in the reactor would have meant a much greater modelling effort and longer computation times, which would have meant limiting the investigation to only a section of the reactor.

With the Euler-Euler method each phase (here air and algae fluid) is assumed to be continuous and is modeled by averaged conservation equations. In the process of the mathematical averaging, the phase fraction is defined which represents the probability that each phase can be found in any cell in the mesh at a certain time (Hill 
1998), (Rusche 2002). The VoF method integrates a coupling between the phases in both directions and is a standard approach for the industrial simulation of twophase flows, especially for higher phase fractions (Rusche 2002).

Within the context of the transient CFD simulations, the algae fluid phase is modelled in a simplified fashion and assumed to have the physical characteristics of water, since the microalgae have a negligible influence on the flow properties of the fluid with the normal algae concentrations occurring during growth in the reactor (Hindersin et al. 2013), (Leupold et al. 2013). In the case that the algae concentration in the system would become high enough to significantly increase fluid viscosity, it would also become a problem for the circulation pumps in the system (that transport the algae fluid between the PBRs and the heat exchangers in the building). Regular harvesting of the microalgae prevents this and ensures that the viscosity of the algae fluid remains practically the same as that of water.

In addition, the small volume flow of the $\mathrm{CO}_{2}$ supplied into the reactor (to allow for algae photosynthesis) and the inflow and outflow of the algae fluid into the heat exchanger are not taken into account because the resulting velocities are about an order of magnitude smaller than the velocity gradients generated by the airlift injections.

It should be emphasized that the aim of this investigation is to compare different reactor geometries with regard to the magnitudes and variances of the flow velocities in the reactor. It is not the goal to simulate the two-phase flow characteristics as accurately as possible with a completely resolved phase boundary of the bubbles as they rise.

The two-phase flow simulations are performed with the solver twoPhaseEulerFoam, which was developed for gas-liquid flows with disperse gas bubbles in a liquid (Greenshields 2015), (Marschall 2011). This solver uses the PIMPLE algorithm, which is a combination of the common algorithms SIMPLE (by Patankar) and PISO (by Issa) (Greenshields 2015), (Patankar 1980), (Issa 1985).

Structured calculation meshes of approx. 0.7 million cells per reactor are generated for the simulations using the OpenFOAM utility blockMesh. The reason for this comparatively small number of cells is the highly dynamic behavior of the flow. This results in the necessity of a very small time step in the transient simulations, which leads to a correspondingly long calculation times and requires a limitation of the mesh size.

The simulations are carried out isothermally, as no large temperature gradients occur within the PBR during typical operation. The two fluids air and algae fluid are modelled as immiscible phases without any changes in chemical composition. The biological processes of the algae cells during growth are not modelled in the modelling and simulations. Thus, no reactions or phase interactions occur, except for impulse exchange or interphase impulse transport.

In order to determine the predominant flow regime (laminar/turbulent) in the reactor for the simulations, the two-phase Reynolds number $\operatorname{Re}_{U m}$ (with $U_{m}$ signifying the average velocity between the two phases, see equation 1) is assessed and compared with empirical results in the literature.

In equation $1, v_{m}$ signifies the two-phase kinematic viscosity according to Dukler (see equation 2) and $D_{h}$ the hydraulic diameter of the reactor channels (Dukler et al. 1964). In equation 2 the kinematic viscosity of the respective phases is weighted by the corresponding mass fraction $x_{i}$

$$
\operatorname{Re}_{U_{m}}=\frac{D_{h} U_{m}}{v_{m}}
$$

The reactor is filled to more than $90 \%$ of the volume with algae fluid (fill level $2.8 \mathrm{~m}$ with a reactor height of $3.0 \mathrm{~m}$ ), however the mass of the fluid is much greater than the air which means that the phase weighted twophase kinematic viscosity is then roughly equal to the kinematic viscosity of the algae fluid (modelled here as water)

$$
v_{m}=\left(1-x_{\text {gas }}\right) v_{\text {liquid }}+x_{\text {gas }} v_{\text {gas }}
$$

Assessing the experimental flow velocities in test reactors documented in Leupold et al. 2013, an average flow velocity of $0.2 \mathrm{~m} / \mathrm{s}$ is assumed, which results (using equation 1) in a two-phase Reynold number of 3880 . This value characterises the flow as being in the transition zone according to several authors (i.e. Clift et al. 1978, Ratkovich 2009, Wang 2015), however not yet fully turbulent. Experimental results recorded in the literature indicate a turbulent regime developing fully at two-phase Reynolds numbers of 4000-5000 (Clift et al. 1978), (Ratkovich 2009), (Wang 2015).

In viscous liquids the kinetic energy can be completely dissipated by laminar viscous dissipation, while in a fluid with a slightly lower viscosity (such as water) the wake of a bubble can be stirred up while the flow regime still remains laminar (Rusche 2002). Bubble movements are often transient and very complex; fluctuations of the bubble movement in several directions are thus often also superimposed with changes of shape and oscillations (Rusche 2002). In this respect, irregular bubble movements can also occur at low flow velocities and in laminar flows or flows in the transition area. The dynamic bubble movements themselves can also cause small amounts of local flow agitation in the bubble wake in an otherwise laminar flow which is characterized as so-called 'bubble-induced turbulence' (Lance and Bataille 1991).

Corresponding to these considerations and the two-phase Reynolds number calculation, the flow in the simulations is modelled with a laminar regime. 


\section{Results and Discussion}

In this section a selection of simulated reactor variants and their results are presented and discussed. An overview of the reactor variants presented can be found in Table 1. Images and characteristic details of each of the reactor variants are summarized in Table 2 . Within the framework of the analysis of the results, plots of the averaged vertical velocities (over the reactor height) as well as transverse velocities over the reactor depth (the so-called 'horizontal velocities') over the height of the reactor are presented for each selected variant. Images of the simulation results are not used for the evaluation; however, two exemplary snapshots of the velocity and phase fraction for variant B are shown in figures 5 and 6 to give an impression of the visual results.

Table 1: Selection of reactor variants studied

\begin{tabular}{|c|c|}
\hline Variant & Description \\
\hline Var A & Short channel dividers (2.0 m high) \\
\hline Var B & Long channel dividers (2.6 m high) \\
\hline Var C & No channel dividers (point supports 0.05 m high) \\
\hline Var D & Long channel dividers with gap in the middle \\
\hline
\end{tabular}

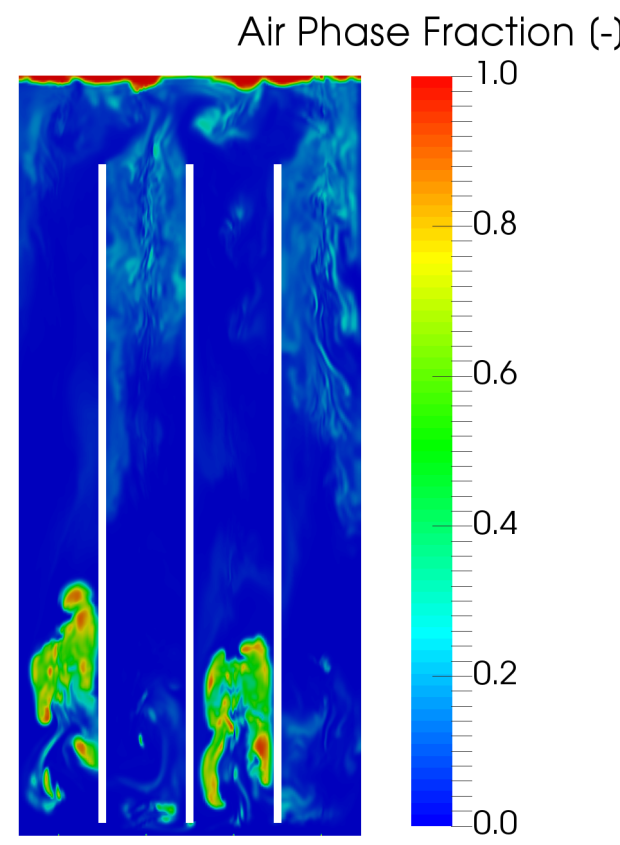

Figure 5: Air phase fraction in simulated reactor variant Var B one second after air injection

\section{Variant A}

Figure 7 shows the velocity plots of variant A averaged over time for an airlift injection cycle. A comparison of the velocity profiles and the simulation images shows that the bubbles rise faster in the central channels than in the side channels.

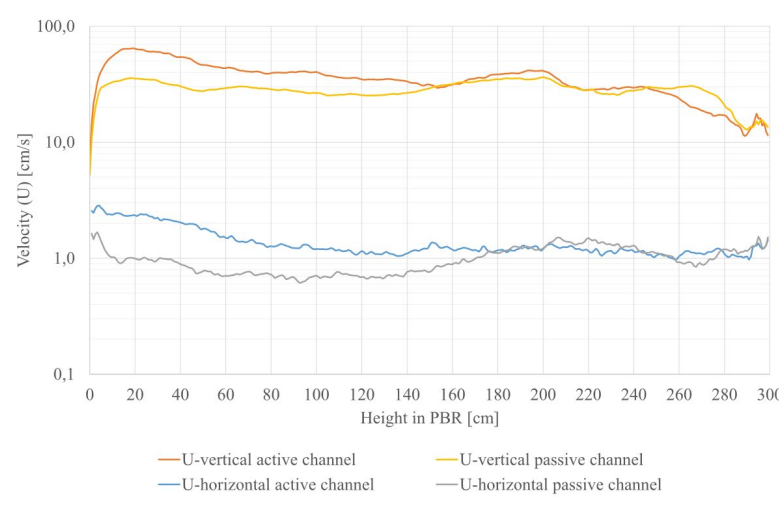

Figure 6: Averaged fluid velocities across depth of reactor for variant Var $A$

This behavior is due to the fact that the middle columns are each connected to two other channels via the connecting openings at the bottom of the reactor. This means that the algae fluid can flow faster from the adjacent channels than from the side channels, which each have only one adjacent channel. The facilitated flow of the algae fluid from the adjacent channels means that the displacement effect of the air bubbles occurs more quickly, meaning that they can also rise faster. This effect is particularly noticeable with the vertical velocities of the passive channels (channels without air injection during an airlift cycle) and in the top section of variant $\mathrm{A}$.

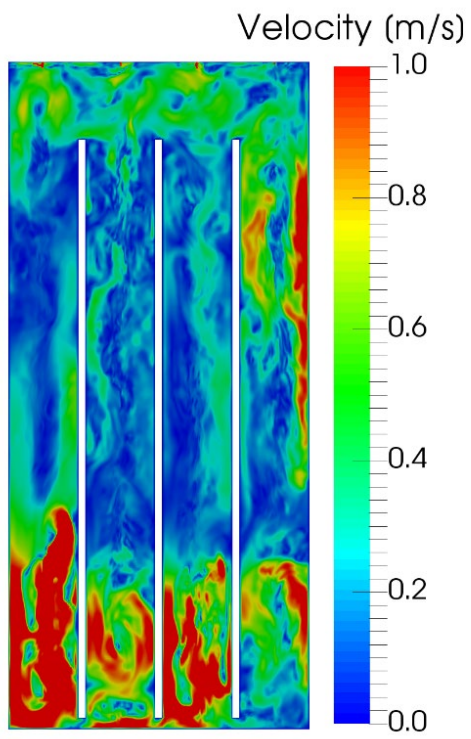

Figure 7: Fluid velocities in simulated reactor variant Var $B$ one second after air injection

\section{Variant B}

Figure 8 shows the simulation results of variant $\mathrm{B}$. The comparison of the velocity profiles over the reactor height doesn't allow any clear qualitative conclusions with regard to the superiority of the simulation variants $\mathrm{A}$ and $\mathrm{B}$, as the profiles are so similar. A more detailed assessment of the velocities in the reactor can be found in the statistical evaluation of all cells in Figure 11. The reason for the small but significant statistical difference 
in the velocities is the length of the dividers. Contrary to expectations that a large flow circulation in the top section of the reactor would have favourable effects on the flow velocities, the results of variant B show that the dividers also favour sustained velocity gradients in the upper half of the reactor stemming from the airlift injections.

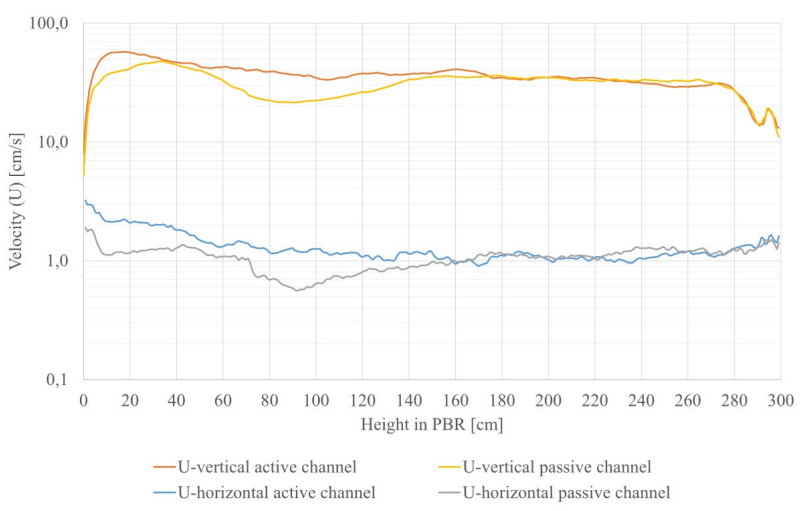

Figure 8: Averaged fluid velocities across depth of reactor for variant Var $B$

Since the movement of the algae fluid is restricted by the dividers, the impulse of the compressed air injection and the associated fluid displacement by the air bubbles ensures a faster flow of the fluid particles in the reactor channels. This effect is particularly noticeable with the vertical velocities of the passive channels (channels without air injection during an airlift cycle) and in the top section of variant $\mathrm{A}$.

\section{Variant C}

In contrast to variants $\mathrm{A}$ and $\mathrm{B}$, variant $\mathrm{C}$ has no dividers; only $5 \mathrm{~cm}$ high point supports are used to brace the glass panels. The resulting reactor model therefore has no division into separate channels and the algae fluid can flow freely in the reactor.

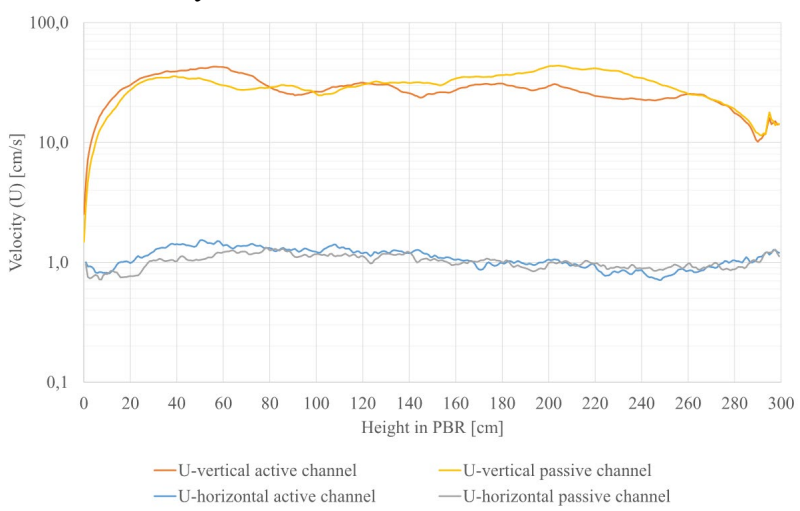

Figure 9: Averaged fluid velocities across depth of reactor for variant Var $C$

The velocities of the variant $\mathrm{C}$ are somewhat lower (see figure 9), which can be seen from the comparison with variants $A$ and $B$. As each of the air injections is distributed across the entire fluid volume of the PBR, the velocity gradients in the reactor in variant $\mathrm{C}$ are generally lower. Furthermore, there is no limitation of the flow by the dividers, which have the positive effect of concentrating and sustaining the fluid velocities for longer periods (as is strongly pronounced in variant B). Instead of higher velocities, however, the flow of variant $\mathrm{C}$ is characterized by more flow circulation and mixing.

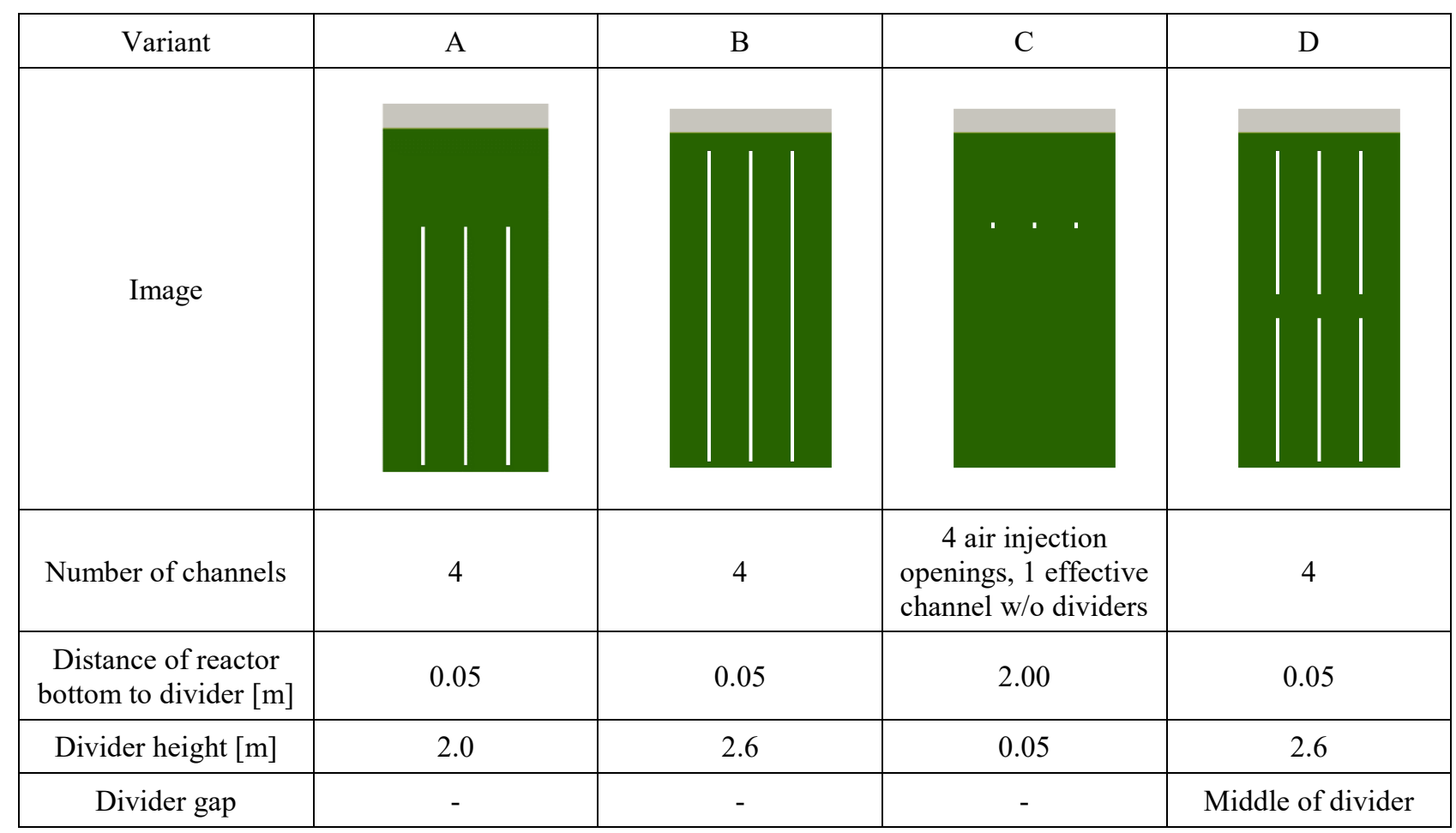

Table 2: Overview of simulation variants described 


\section{Variant D}

The simulated velocities of variant D are presented in Figure 10. The velocity profiles clearly show that a trade-off in the magnitude of the velocities occurs in the upper half of the reactor due to the divider gaps.

Due to the induction effect of existing velocity gradients of the adjacent channels, the air bubbles of the active channels (with air injection in that particular airlift cycle) are partly drawn into the passive channels (without air injection in that particular airlift cycle). The suction effect of the passive channels (particularly pronounced in the middle channels) causes an uneven flow through the upper channel halves.

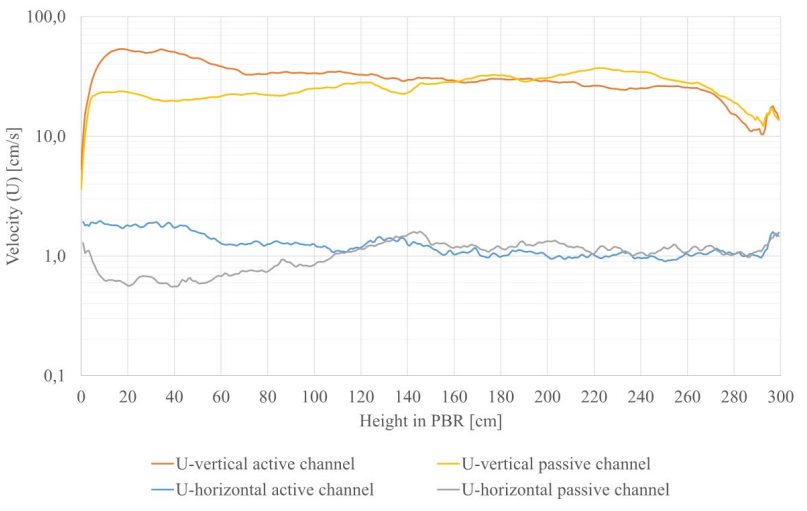

Figure 10: Averaged fluid velocities across depth of reactor for variant Var D

\section{Comparison of Variants}

In the final step of the analysis, all variants are statistically evaluated by combining and averaging all the values calculated in all cells over several time steps in order to obtain a simple comparison of the variants. As the transverse velocities over the reactor depth are of particular importance for the growth of the algae (see Concept section), a comparison of these velocities is of interest to determine the most suitable reactor geometry for promoting microalgae growth.

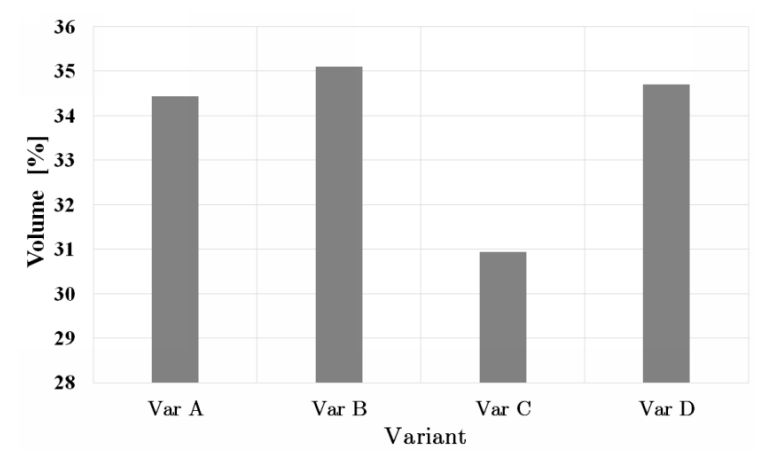

Figure 11: Comparison of amount of reactor volume with average horizontal fluid velocities greater than

$1 \mathrm{~cm} / \mathrm{s}$ across the reactor depth for simulated variants

Figure 10 shows the results of the statistical analysis, which compares the mean volume fraction with transverse velocities greater than $1 \mathrm{~cm} / \mathrm{s}$. (The larger the volume with velocities $>1 \mathrm{~cm} / \mathrm{s}$, the more efficient the growth of the algae in the reactor). From the results of this analysis it can be concluded that variant $\mathrm{B}$ has the highest transverse velocities on average and is therefore selected as the preferred variant.

Variant B also has the structural advantages of longer and continuous dividers. (The separators serve as supports for the glass surfaces which are exposed to strong pressure fluctuations when the compressed air is injected into the reactor).

\section{Model Validation}

During this study, a prototype of variant B was produced for test purposes, which was used for a rough model validation. Camera recordings of the algae fluid flow resulting from the air injection into the prototype (reactor variant B) were made and then divided into individual images. A finely divided optical grid raster is superimposed onto all the images so that the movements of the air bubbles and fluid particles can be tracked (see figure 12).

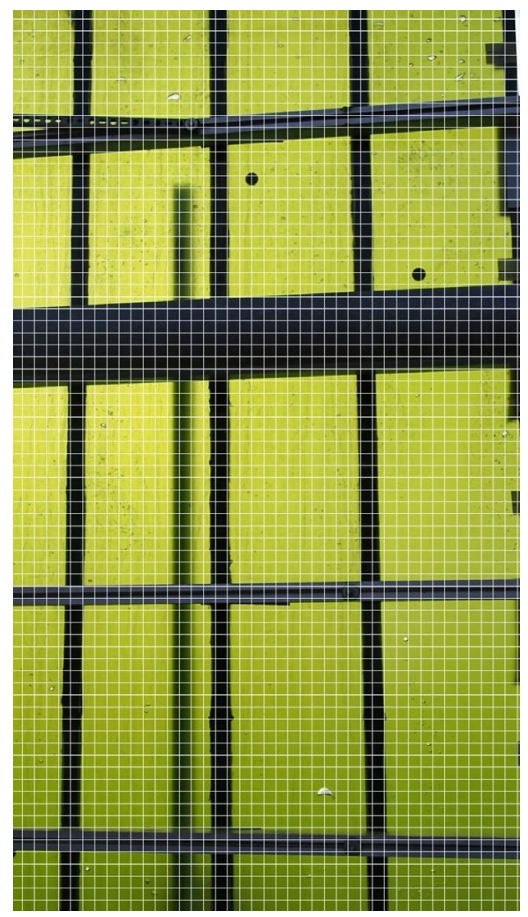

Figure 12: Example still image from a flow recording with optical raster used to track bubbles and particles.

By following small bubbles and particles in the flow, rough velocity profiles in the reactor can be determined. This approach neglects however the velocity gradients over the depth of the reactor and attempts rather to benchmark the velocities over the height and width. Collating the optically tracked velocities in the reactor prototype results in an estimation of the fluid velocities in the reactor channels, which can then be compared 
with the transient output from the flow simulations Approximate velocity profiles in the reactor at a height of approx. $1 \mathrm{~m}$ in the middle of channels 2 and 4 are compared with the calculated values from the simulations as shown in Figures 13 and 14.

The tendency of the simulation results roughly follows the that of the data gathered from the optical measurements, however strong fluctuations in the absolute values are present with deviations of up to around $100 \%$ of the values studied, which can be due to the resolution of the mesh chosen as well as the size of the time steps (smaller more conservative time steps might be preferable in this case). Furthermore, the computation model used (VoF) to simulate the air phase without actually resolving the interface between fluid and gas likely also plays a significant role in the discrepancies observed.

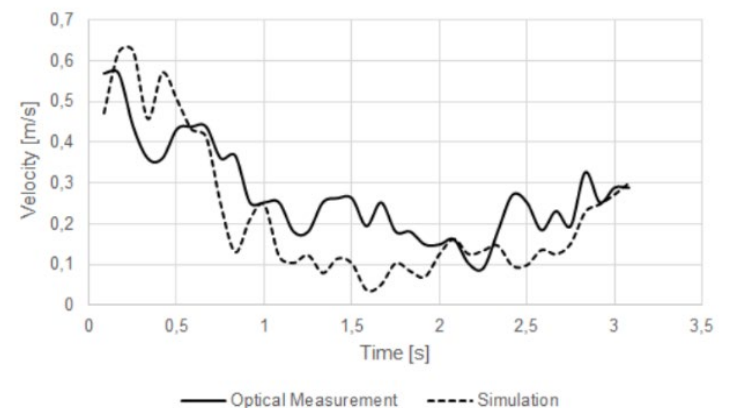

Figure 13: Comparison of simulation results of Var B (middle of channel 2) with optical camera measurements of algae fluid flow in reactor prototype

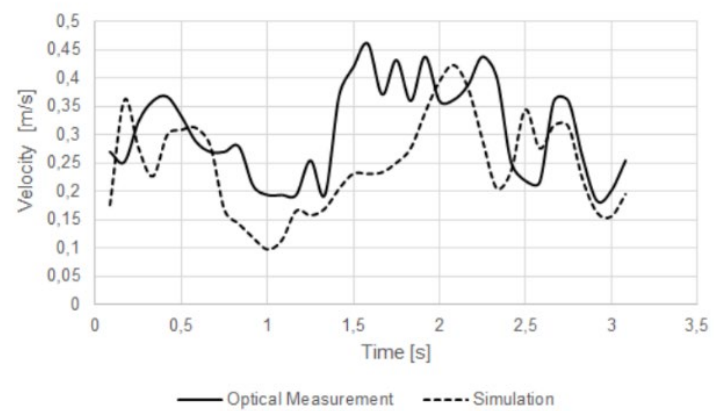

Figure 14: Comparison of simulation results of Var B (middle of channel 4) with optical camera measurements of algae fluid flow in reactor prototype

The goal of the simulation analysis is to however estimate which geometries are beneficial for the development of higher velocities in the reactor not to exactly predict the real velocities. As a result, the goal of this preliminary validation is to assess if the order of magnitude of the velocities being calculated in the reactor is reasonable. Fluctuations of $+/-100 \%$ in the absolute velocity measured are less critical if the overall tendency of the flow velocities in each of the channels is similar.

As described in the Methods section on page 3, bubble movements are very dynamic and complex; this together with the local phenomenon of bubble-induced turbulence makes them challenging to model computationally even if one were to use a very fine grid with individually resolved bubbles. Such an approach is however currently still too computationally expensive to be feasible for use in computational design analysis.

The estimation of average velocities is sufficient for this application and this first model validation indicates a satisfactory comparison against the simulation results for the purpose of this study. Nevertheless, it is clear from the deviations between the data sets that the agreement with experimental results could be improved. For this reason, more detailed stereo PIV measurements of the reactor prototype are planned in the future to better assess the three dimensional velocities in the reactor and further calibrate the computational model based on the results.

The next steps in the research project encompass the further development and testing of the reactor as well as the determination of a suitable approach for PIV measurement of the entire PBR.

\section{Conclusion}

With the aid of flow simulations conducted with the CFD software OpenFOAM, different reactor variants are investigated with regard to their flow characteristics in order to select an improved reactor design for more efficient façade-integrated photobioreactors.

Simulations of several variants are carried out in order to assess the flow behaviour in each simulation variant. The development of the velocity gradients over the reactor height as well as the numerical values of the cells in the reactor model are evaluated. Through variations of the height and characteristics of the dividers in the reactor (see summary in table 2), a PBR geometry is identified which favours the formation of high horizontal velocity gradients in the algae fluid phase and at the same time shows a robust static behaviour with regard to structural stresses and loads.

A first model validation of velocities in a preliminary reactor prototype shows a satisfactory agreement between the calculated simulation values and the velocities in the reactor.

\section{References}

Becker, S., Bie, H. D. and J. Sweeney (1999). Dynamic flow behaviour in bubble columns. Chemical Engineering Science 54, 4929-4935.

Clift, R., Grace, J.R., Weber, M.E. (1978). Bubbles, Drops, and Particles. Academic Press. New York (USA).

Dukler, A., Moye, W. and R. Cleveland (1964).

Frictional pressure drop in two-phase flow. Part A: a comparison of existing correlations for pressure loss and holdup, and Part B: an approach through similarity analysis. American Institute of Chemical Engineers Journal 10, 38-51 
Greenshields, C.J. (2014). OpenFOAM - User Guide Version 2.3.1. OpenFOAM Foundation.

Greenshields, C.J. (2015). OpenFOAM - Programmers Guide - Version 3.0.1. OpenFOAM Foundation.

Hill, D.P. (1998). The Computer Simulation of Dispersed Two-Phase Flows. Dissertation. University of London (UK).

Hindersin, S., Leupold, M., Kerner, M. and D Hanelt (2013). Irradiance optimization of outdoor microalgal cultures using solar tracked photobioreactors. Bioprocess Biosystems Engineering 36, 345-355.

Hindersin, S., Leupold, M., Kerner, M. and D. Hanelt (2013). Key parameters for outdoor biomass production of Scenedesmus obliquus in solar tracked photobioreactors. Journal of Applied Phycology 26, 2315-2325.

Issa, R. I. (1985). Solution of the Implicit Discretized Fluid Flow Equations by Operator Splitting. Journal of Computational Physics 62, 40-65.

Lance, M. and J. Bataille (1991). Turbulence in the liquid phase of a uniform bubbly air-water flow. Journal of Fluid Mechanics 222, 95-118

Leupold, M., Hindersin, S., Kerner, M. and D. Hanelt (2013). The effect of discontinuous airlift mixing in outdoor flat panel photobioreactors on growth of Scenedesmus obliquus. Bioprocess Biosystems Engineering 36, 1653-1663.

Marschall, H. (2011). Towards the Numerical Simulation of Multi-Scale Two-Phase Flows. Dissertation. Technical University Munich (DE).

Patankar, S. V. (1980). Numerical Heat Transfer and Fluid Flow. Mcgraw-Hill. Washington (USA).

Ratkovich, N. (2009). Experimental study and CFD modelling of a two-phase slug flow for an airlift tubular membrane. Chemical Engineering Science $64,3576-3584$

Rusche, H. (2002). Computational Fluid Dynamics of Dispersed Two-Phase Flows at High Phase Fractions. Dissertation. Imperial College London (UK).

Wang, Y. (2015). Slug flow in a vertical narrow rectangular channel - Laminar and turbulent regimes in the main flow and turbulent regime in the wake region of the Taylor bubble. Progress in Nuclear Energy 85, 164-177

Ziegenhein, T., Rzehak, R. and D. Lucas (2014). Transient simulation for large scale flow in bubble columns. Artikel-Preprint to Chemical Engineering Science. Helmholtz-Zentrum Dresden-Rossendorf (DE). 